\title{
NOMBRE, AUTORIDAD Y EL SISTEMA DE CREENCIAS EN LOS ALTOS DE CHIAPAS ${ }^{1}$
}

\author{
Por Roberta Montagu y Eva Hunt. \\ Universidad de Chicago.
}

Las hipótesis presentadas en esta ponencia se refieren a la correlación de tres clases de información etnográfica que parecen estar interrelacionadas por participar de un solo sistema lógico.

El primer grupo de datos se basa en un sistema organizado para dar nombre a las personas, el sistema de jelol, ${ }^{2}$ que puede ser dividido en dos tipos: el jelol genealógico y el jelol posicional.

Aunque la palabra más aproximada para traducir jelol es tocayo, el concepto de jelol denota que el tocayo de una persona es "su reposición en la tierra", lo que sucede por medio de la adquisición del nombre.

Un jelol genealógico es una persona a la que se le ha dado el nombre de un pariente en la generación ascendente. Es más común, no obstante, que esta forma de jelol se limite a los parientes de un individuo dentro del grupo de descendencia agnática. En este caso, tanto la persona como su "reposición" comparten el nombre y el apellido o apellidos. Es así, que si el jelo! de un hombre es el hijo del hermano, los dos compartirán el mismo nombre como una unidad (los dos se llamarán, por ejemplo, Hpetul Hsiwnes Huacax), mientras que si su jelol es

1 Deseamos agradecer a los siguientes trabajadores de campo el habernos hecho disponibles sus materiales aún no publicados: Frank y Francesca Cancian, Marcelo Díaz de Salas, Calixta Guiteras H., Esther Hermitte, Andrés Medina, Duane y Barbara Metzger, Manning y June Nash, Henning Siverts y Manuel Zabala.

Hemos además consultado los materiales publicados sobre la región por Calixta Guiteras H., Ricardo Pozas, Henning Siverts y Alfonso Villa Rojas.

2 En esta ponencia hemos usado la palabra tzeltal jelol. La palabra, pero no el concepto, varía; por ejemplo, en Zinacatán y Chamula la palabra es squ'exol. 
el hijo del hijo de la hermana, solamente compartirán el nombre de pila (uno será Hpetul Hsiwnes Huacax, el otro Hpetul Mentes Ton). Entre los tzeltales de las fincas de la región de Ocosingo, existe un sistema de nombres aún más complejo, en que varios apellidos cambian como jelolil dentro de un mismo grupo agnático, tema que será discutido en una ponencia separada.

Un jelol posicional es una persona que sucede a otra en su cargo, tanto en el cuerpo religioso como en el cuerpo polítieo que forman el gobierno jerárquico de los poblados indígenas. La duración de un cargo es generalmente de un año, y un oficial público selecciona a su propio jelol, aunque en algunas comunidades la selección de todos los jelolil es hecha por uno o más de los oficiales que tienen esta responsabilidad específica. Cada oficial o "carguero" tiene un título, y en cuanto toma el cargo adquiere el nombre de ese título. En Tenejapa, por ejemplo, el hombre que funciona como Primer Alférez de la Fiesta de la Virgen, es llamado Banquilal Captán yu'un Jalame'tic.

Una diferencia básica entre los dos tipos de jelolil es que el primero puede incluir más de dos individuos: en muchos casos, varios miembros del grupo agnático comparten el mismo nombre y son jelolil, contemporáneamente. En Sivacá, por ejemplo, varios hermanos, incluso, llevan el mismo nombre y apellido. En el otro caso, un oficial es el jelol de su predecesor y él mismo tendrá su jelol o reposición, pero esta relación nunca incluye más que dos individuos en un marco vertical.

La segunda clase de información etnográfica pertinente, atañe a las creencias sobre el alma o almas, y el concepto adjunto de c'al (literalmente "calor del alma", pero una traducción más apropiada sería "potencia del alma").

Los indígenas tzeltales y tzotziles creen que un individuo posee almas múltiples. El número y las características particulares de estas almas varía de grupo a grupo. De estas almas sólo de dos puede decirse que su creencia está generalizada en el área: un alma inmortal que reside en el cuerpo y lo abandona a la muerte (la que no será discutida en esta ponencia), y un alma animal, de una especie determinada, que reside en lugares sagrados, en los cerros que rodean a los pueblos. El alma animal y la persona se consideran separadas pero son una al mismo tiempo, porque comparten la misma "esencia vital", 
aunque la identidad del espíritu animal no es necesariamente conocida por su "dueño". La participación de la misma esencia vital se manifiesta en la participación de:

1. La salud. Si el alma animal es herida, el cuerpo humano refleja el golpe; si lo matan, la persona muere. Muchos síntomas de enfermedades se explican en estos términos. Si una persona descubre un moretón que no recuerda haber sido causado por un golpe, dice que "fue mi ch'ulel (alma animal) ${ }^{3}$ que se golpeó".

2. La fuerza. El niño nace junto con su alma animal. Cuando el animal crece en edad y fuerza física, el niño llega a ser más fuerte. Ciertas especies animales tienen mayor fuerza física que otras, y de la gente que tiene más fuerza que sus semejantes se dice que tiene esta clase de alma animal.

3. Características físicas. Una persona que se parece a cierto animal en algún rasgo físico se cree que tiene a este animal por su contraparte anímica. Para una persona que tiene mucho pelo en el cuerpo, por ejemplo, se cree que su alma es un mono.

4. Persondidad. Se supone que ciertos rasgos de personalidad son manifestaciones de un alma animal particular, tal como astucia de alma del coyote, o suavidad de alma del venado. A un brujo se le considera como teniendo alma de tigre; esta asociación se puede explicar por el hecho de que se considera a ambos como comedores de seres humanos: el tigre de la carne, y el brujo del alma animal.

La esencia vital es considerada un bien escaso, infinitamente divisible. Hay una cantidad limitada tanto para el individuo como para el grupo. En consecuencia, cuanto más se comparte, menos asequible es. Los individuos así como los grupos compiten entre sí por su posesión. Una persona puede perder parte de su esencia vital así como adquirir más. Cuanto más esencia vital acumula una persona, más aumenta la potencia de su alma. Dos criaturas que nacen en la misma vecindad compiten por la esencia vital, y se dice que una morirá y la otra crecerá lozana. Este concepto se extiende dentro de otros dominios de la vida del indígena, tal como la agricultura. Se considera que el maíz tiene un alma que puede ser robada o debilitada. Un

3 El alma animal recibe diversos nombres a través del área; en Chamula es el wayojel, en las fincas es llamada wayjel, en Oxchuc y Cancuc es el lab, en Zinacantán y Pinola es el ch'ulel. 
hombre puede hacer una transmisión de la fuerza o potencia de una milpa ajena a la suya. Una comunidad puede hacer lo mismo con las milpas de otra comunidad.

Un individuo puede acumular esencia vital y por lo tanto aumentar su potencia de alma por medios legítimos e ilegítimos. Los medios legítimos son a través del proceso natural de sobrevivir a otras personas, y sirviendo a su comunidad en cargos específicos. El método ilegítimo es el de robar la esencia de vida de otras gentes por medio de varias técnicas de brujería, de las cuales la básica es obtener almas animales que pertenecen a otros.

Los jelolil genealógicos, en la mayor parte de las comunidades de los Altos, son considerados como compartiendo la misma esencia vital y un alma animal de la misma especie. El nombre de la persona también se identifica estrechamente con la esencia vital de la persona. Las siguientes prácticas corresponden a esta creencia, aunque no ocurren necesariamente en todas las comunidades:

1. Antes de que a una criatura se le dé un nombre, en una ceremonia nativa o en el bautismo de la iglesia, se considera que el alma no está atada a la persona, por lo que una vigilancia estrecha se ejerce sobre el recién nacido de parte de los miembros de su grupo doméstico, para evitar que la esencia de vida se escabulla del cuerpo. En Tenejapa es común esta práctica.

2. Entre los indígenas de las haciendas de Ocosingo, el padre de la criatura sueña o "siente en su corazón" que su hijo tiene la misma alma animal de otro individuo de su grupo agnático. La criatura, por lo tanto, llevará el mismo nombre de este otro $\mathrm{u}$ otros individuos, puesto que si han nacido con la misma especie de alma animal, han "nacido con el mismo nombre". Esta práctica contradice otra que se basa, sin embargo, en la misma creencia:

3. En Tenejapa, a un infante no se le da el nombre de un jelol mientras el "dueño" original del nombre esté vivo, porque se piensa que el menor, junto con el nombre, adquirirá la potencia de alma del mayor, cosa que causará la muerte del adulto.

4. A lo largo de toda la región de los Altos, un individuo tiene un nombre secreto, con el propósito de proteger el alma animal con la que comparte la esencia de vida, siendo así que 
se "defiende" la potencia de alma individual de los ataques de brujería.

Para proteger la potencia del alma de la milpa, se siguen en toda la región ciertas prácticas rituales, tanto en la iglesia, como en el maizal y el granero, al plantar, durante la cosecha, y en otras ocasiones semejantes.

El tercer grupo de datos etnográficos se relaciona con los mecanismos por medio de los cuales una persona adquiere autoridad.

Durante el curso de la vida de un individuo, éste tiene la posibilidad de llegar a ser jelol posicional y luego oficial de cargos políticos y/o religiosos que tienen nombres específicos y otorgan al poseedor títulos determinados. Durante su período como oficial, la persona es llamada por su título en ciertos contextos, por ejemplo, al ser saludada en la calle, dentro del Cabildo (el edificio de gobierno local) y en ocasiones ceremoniales. Después de que ha terminado su mandato, el título-nombre aún le pertenece, prefijado por la palabra pasaró (pasado). Así es que cuantos más puestos oficiales el individuo sirve, más títulos o nombres colecciona.

$\mathrm{La}$ disponibilidad de posiciones oficiales varía de comunidad a comunidad en una proporción inversa entre el número de posiciones y el monto de la población. En Aguacatenango, con una población de aproximadamente 800 habitantes y más de 30 cargos, prácticamente cada adulto varón, de acuerdo con su grupo de edad, ha pasado los cargos normativos. En Zinacantán, sin embargo, con 53 cargos oficiales y una población de 7,600 , muy pocos adultos pasan por todos los cargos de su grupo de edad. Esto, por sí mismo, denota que existe competencia para la acumulación de títulos por individuos del mismo grupo de generación. Existe una correlación definitiva entre la competencia para la acumulación de nombres y la competencia para adquirir la esencia vital, es decir una rivalidad para obtener poder natural o sobrenatural.

Durante el proceso en que obtiene éxito en la acumulación de nombres, la persona envejece. Estos dos factores combinados (el envejecer y el adquirir nombres-títulos) le dan a la persona creciente prestigio y un aumento de su autoridad frente la comunidad.

El aumento de poder personal dentro de la sociedad se puede explicar, al nivel metafísico, de la siguiente manera. 
1. Se cree que una persona anciana tiene más conocimientos y merece mayor respeto y obediencia porque: a) al haber sobrevivido a otras gentes ha acumulado para sí misma más de la potencia de alma disponible del fondo general de esencia vital; b) ha resistido los ataques de brujería, tanto planeados como involuntarios, dirigidos en contra de su alma animal; por lo tanto ha demostrado la potencia de su alma. Solamente las almas fuertes se consideran aptas para resistir la brujería. Los infantes, con sus delicadas almas, son las víctimas más fáciles de la brujería, que se dirige hacia ellos, indirectamente, cuando se quiere dañar a los padres, los que por ser adultos pueden "resistir mejor" los ataques. De esta manera se explica, entre otras cosas, la alta mortalidad infantil.

2. Una persona de la que se piensa que tiene gran potencia de alma es considerada con poder para controlar otras almas menores, teniendo así autoridad sobre los "dueños" de estas almas. El individuo, de esta manera, mantiene su autoridad, amparado en sus atributos sobrenaturales.

3. Se piensa que los nombres en sí mismos tienen esencia vital, y que la acumulación de nombres incrementa la potencia de alma del dueño de los nombres. Esto refuerza el aumento natural de potencia de alma obtenido mediante el aumento en edad, y solidifica la autoridad al nivel secular.

Un hombre que ha alcanzado una alta posición en el sistema jerárquico de autoridad es una persona que es temida, aunque se le considere un buen individuo. Podemos indicar que a una persona que tiene autoridad sobre otras, se acercan a ella con sentimientos contradictorios ya que "de todos modos, el hecho de la autoridad parece convertir la relación en ambigua, o, como diría el psicoanalista, ambivalente" (Homans, George, 1950:244). Este hecho sociológico está elaborado por los indígenas dentro del sistema de creencias, del siguiente modo:

$\mathrm{Al}$ acumular potencia de alma por medios legales, y el poder y autoridad secular que la acompañan, se deja menos de este bien, escaso de por sí, para el resto de la población, apropiándose, por lo tanto, de un bien común. Además, como se ha mencionado, una persona puede incrementar su potencia de alma por medio de las técnicas ilegítimas de la brujería. Así, cualquier individuo considerado con gran potencia de alma, puede ser sospechoso, y es frecuentemente acusado, de 
ser brujo. El hombre que ha aumentado su prestigio y autoridad sirviendo legítimamente a la comunidad como oficial, puede ser tachado de brujería y asesinado. Esto ocurre con frecuencia, siendo el caso clásico el de Oxchuc en donde el oficial más alto en la jerarquía, el c'atinab, es casi siempre asesinado "por brujo". Se deduce entonces que todo poder, incluso aquel derivado de fuentes legítimas temporales, es clasificado ambiguamente, como originado tanto en fuentes sobrenaturales buenas como malignas.

De esta presentación se pueden derivar las siguientes proposiciones: a) las variaciones en prácticas rituales particulares no son contradictorias, sino alternativas de conducta, encadenadas al mismo sistema metafísico, común en toda la región; b) el sistema de creencias es una parte integral de la vida de la sociedad indígena, que afecta las acciones diarias; c) las ambigüedades en el sistema de creencias no ocurren arbitrariamente, ni al azar, pero reflejan ambigüedades equivalentes o puntos de conflicto en parte de la estructura de la sociedad, en este caso, en el sistema de autoridad.

\section{REFERENCIA BIBLIOGRAFICA}

Homans, George: The Tuman Group. 1950, p. 244.

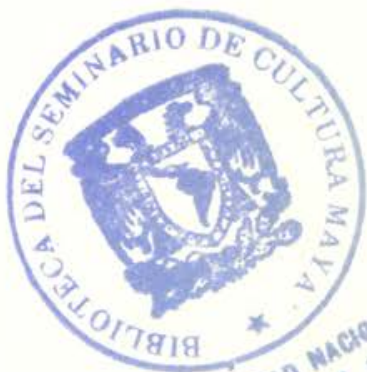

